\title{
Leptocorticium gloeocystidiatum sp. nov. (Basidiomycota), a new corticioid fungus from Sicily, Italy
}

\section{Gorjón SP ${ }^{1}$ and Saitta $A^{2}$}

${ }^{1}$ Universidad de Salamanca. Salamanca, España 37007.
${ }^{2}$ Dipartimento di Scienze Agrarie e Forestali, Università di Palermo. Palermo, Italia I-90128.

Gorjón SP, Saitta A 2014 - Leptocorticium gloeocystidiatum sp. nov. (Basidiomycota), a new corticioid fungus from Sicily, Italy. Mycosphere 5(3), 406-409, Doi 10.5943/mycosphere/5/3/2

\begin{abstract}
A new corticioid species, Leptocorticium gloeocystidiatum is described from Sicily, Italy. It is characterized by a resupinate, buff-coloured basidiome and microscopically by the presence of filiform leptocystidia, gloeocystidia, dendrohyphidia, and small ellipsoid, smooth basidiospores, non-reacting in Melzer's reagent. The species is compared with closest relatives. A key to the accepted species of Leptocorticium is provided.
\end{abstract}

Key words - cystidia - Fagus - Mediterranean area - wood inhabiting fungi

\section{Introduction}

Leptocorticium Hjortstam \& Ryvarden was erected to accomodate Corticium cyatheae S. Ito \& S. Imai, a species originally described from Japan [三Leptocorticium cyatheae (S. Ito \& S. Imai) Hjortstam \& Ryvarden] (Hjortstam \& Ryvarden 2002). Nakasone (2005) emended the genus adding two species previously classified in Dentocorticium Boidin \& Gilles [Dentocorticium sasae (Boidin, Cand. \& Gilles) Boidin, Lanq. \& Duhem (ELeptocorticium sasae (Boidin, Cand. \& Gilles) Nakasone; $\equiv$ Dendrothele sasae Boidin, Cand. \& Gilles) from France and Dentocorticium utribasidiatum Boidin \& Gilles (三Leptocorticium utribasidiatum (Boidin \& Gilles) Nakasone) from France and Reunion island] and describing Leptocorticium tenellum Nakasone, from South America. Two more species were included in the genus; Leptocorticium capitulatum Hjortstam \& Ryvarden, from Brazil (Hjortstam \& Ryvarden 2005), and Leptocorticium torrendii (Bres.) Ghobad-Nejhad, from Portugal (三Corticium torrendii Bres.) (Ghobad-Nejhad 2009). Therefore, Leptocorticium currently contains six species of resupinate basidiomycetes, characterized by thin basidiomes, monomitic hyphal system with clamped septa, utriform or stalked basidia, subulate leptocystidia, dendrohyphidia, and smooth, non-reacting in Melzer's reagent basidiospores (Nakasone 2005). The species have frequently been found on bamboo stems, petioles of ferns, or similar substrata (Nakasone 2005, Hallenberg 2012). Leptocorticium was provisionally classified in the Corticiales K.H. Larss., on the basis of morphological characters (Larsson 2007) because there is no molecular information of any species in the genus.

A deviating specimen, recently collected in Sicily, is proposed and described below as new in Leptocorticum. It has been collected in a mixed forest on dead wood of Fagus sylvatica L., in a Mediterranean biodiversity hotspot, where beech trees worldwide reach its southernmost distributional area. 


\section{Materials \& Methods}

\section{Macro- and microscopic examinations}

For light microscopy studies, samples were mounted in $3 \%$ potassium hydroxide $(\mathrm{KOH})$, Melzer's reagent (IKI) to determine dextrinoid or amyloid reactions, and $0.1 \%$ cotton blue in $60 \%$ lactic acid (CB) to determine cyanophily of basidiospore walls. Line drawings were made with a camera lucida attachment. All the specimens are deposited in MCVE and SALA.

Leptocorticium gloeocystidiatum Gorjón \& Saitta, sp. nov.

Figs $1-2$ MycoBank 808223

Diagnosis - It differs from other species in the genus by smaller basidiospores $(4-5 \times 2.5-3$ $\mu \mathrm{m})$ and the presence of gloeocystidia.

Type - Italy, Sicily, Messina, Portella Scarno, 1400 m.a.s.1., 14 Apr 2012, on dead wood of Fagus sylvatica, coll. A. Saitta 483 (SALA, holotype; MCVE, isotype)

Etymology - gloeocystidiatum: referred to the presence of gloeocystidia, a distinctive character within the other species of Leptocorticium.

Description - Basidiome resupinate, pruinose, cream to buff, margin abrupt, indistinct, hymenophore smooth to slightly grandinioid under the lens $(10 \times)$ in some areas. Hyphal system monomitic, hyphae with clamps, thin-walled, 2-3 um in diam., hyaline in the subhymenium and yellowish towards the subiculum. Hymenium a palisade of basidia, gloeocystidia, filiform leptocystidia, and dendrohyphidia. Gloeocystidia clavate or more frequently with two or three constrictions, 20-40 $\times 5-6 \mu \mathrm{m}$ with thickened walls, refringent in $\mathrm{KOH}$ (reaction in sulfobenzaldehyde not tested). Leptocystidia filiform, with an apical hyphoid projection up to 100 $\mu \mathrm{m}$ long, but usually shorter ab. 30-50 $\mu \mathrm{m}$, thin-walled, basally widened and some with knobs or small obtuse protuberances, non-encrusted. Dendrohyphidia few, thin-walled, non-encrusted. Basidia clavate, somewhat constricted and stalked, 15-20 × 3-4 $\mu \mathrm{m}$, with four slender sterigmata, basally clamped. Basidiospores ellipsoid, 4-4.5(-5) $\times 2.5-3 \mu \mathrm{m}$, smooth, thin-walled, hyaline, IKI, CB-.

Known distribution - Known only from the type locality in Sicily.

Other material examined - L. tenellum: Chile, Los Lagos, Puyehue National Park, 21 Feb 2010, on Chusquea quila (Poaceae), coll. N. \& L. Hallenberg, S.P. Gorjón, NH 16294 (GB).

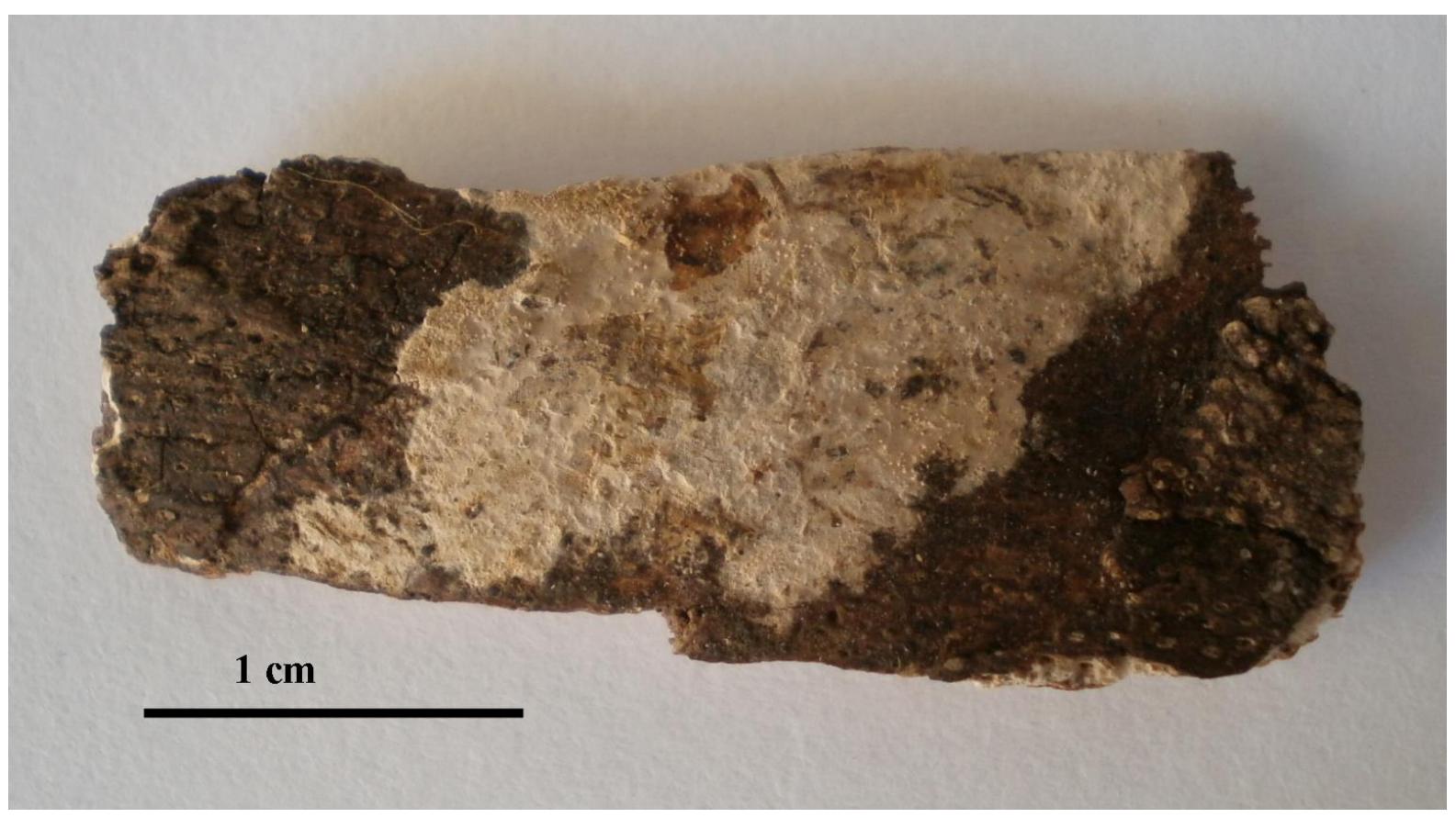

Fig. 1 - Leptocorticium gloeocystidiatum sp. nov. (holotype). Aspect of the basidiome. 


\section{Key to Leptocorticium species}

1. Gloeocystidia present, basidiospores $4-4.5(-5) \times 2.5-3 \mu \mathrm{m}$

L. gloeocystidiatum

1. Gloeocystidia absent, basidiospores longer 2

2. Leptocystidia apically widened and capitate, basidiospores 6-7 $\times 2-2.5 \mu \mathrm{m}$. L.capitulatum

2. Leptocystidia tapering to a subulate apex, basidiospores diverse 3

3. Basidiospores subglobose, 7-8.5(-9.5) $\times 6-8 \mu \mathrm{m}$. L. torrendii

3. Basidiospores narrowly ellipsoid to cylindrical, up to $6-6.5 \mu \mathrm{m}$ wide 4

4. Basidiospores (10-)11-13 × 5-6(-6.5) $\mu \mathrm{m}$, absence of hymenial crystals L. utribasidiatum

4. Basidiospores smaller, usually up $5 \mu \mathrm{m}$ wide, hyaline crystals abundant in the hymenium. 5

5. Basidiospores (7-)8-11.5 × 2.5-3 $\mu \mathrm{m}$, Japan and Reunion island. L. cyatheae

5. Basidiospores wider, more than $3 \mu \mathrm{m}$ wide, with other known distribution 6

6. Basidiospores 8-12(-14) $\times 3.5-5.5 \mu \mathrm{m}$, basidia clavate to suburniform, often with a short stalk, known from Europe L. sasae 6. Basidiospores slightly shorter, 7-9(-11) $\times(3-) 3.5-4.5(-6) \mu \mathrm{m}$, basidia frequently utriform and usually inflated in the base, distributed in South America. L. tenellum

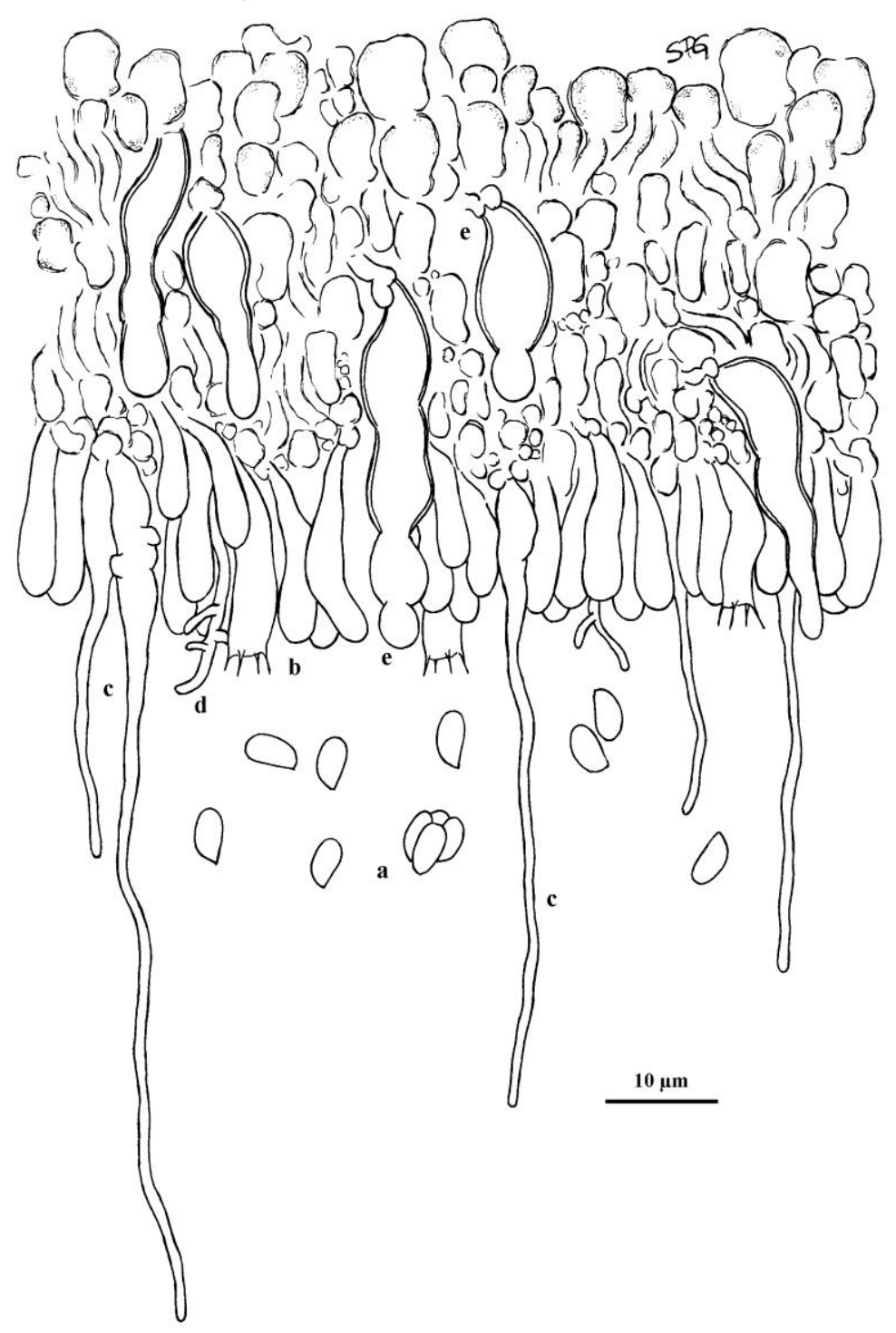

Fig. 2 - Leptocorticium gloeocystidiatum sp. nov. (holotype). Microscopical elements. a, Basidiospores. b, Basidia. c, Leptocystidia. d, dendrohyphidia. e, gloeocystidia. 


\section{Discussion}

Leptocorticium gloeocystidiatum is tentatively described in Leptocorticium because it shares with the rest of the species of the genus common important characteristics, as a thin resupinate basidiome, monomitic hyphal system, clamped hyphae, presence of thin, subulate leptocystidia, dendrohyphidia, and smooth basidiospores not reacting in Melzer's reagent. It deviates from the generic concept by the presence of gloeocystidia and smaller, ellipsoid basidiospores. However, many species of corticioid fungi with gloeocystidia have been added to genera in which the type species lacks such elements (e.g. Aphanobasidium Jülich, Athelopsis Oberw. ex Parmasto, Fibricium J. Erikss., Sistotrema Fr., ett.). Nonetheless, it should be desirable to obtain molecular data of $L$. gloeocystidiatum to test its phylogenetic position and natural relationships.

\section{Acknowledgement}

The critical review and suggestions of an anonymous reviewer is sincerely appreciated.

\section{References}

Ghobad-Nejhad M. 2009 - A new species and new combinations in the genus Gloiothele (Basidiomycota). Mycotaxon 110, 261-270.

Hallenberg N. 2012 - Leptocorticium tenellum (Agaricomycetes) found in Chile. Kurtziana 37(1), $109-112$.

Hjortstam K, Ryvarden L. 2002. - Leptocorticium, a new genus among the corticioid fungi (Basidiomycotina, Aphyllophorales). Synopsis Fungorum 15, 22-25.

Hjortstam K, Ryvarden L. 2005 - New taxa and new combinations in tropical corticioid fungi (Basidiomycotina, Aphyllophorales). Synopsis Fungorum 20, 33-41.

Larsson KH. 2007 - Re-thinking the classification of corticoid fungi. Mycological Research 111, $1040-1063$.

Nakasone KK. 2005 - Leptocorticium (Corticiaceae s.1., Basidiomycota): new species and combinations. Mycological Progress 4(3), 251-256. 